\title{
Coherent method for detection of gravitational wave bursts
}

\author{
S. Klimenko, I. Yakushin†, A. Mercer, G. Mitselmakher \\ University of Florida, P.O.Box 118440, Gainesville, Florida, 32611, USA \\ †LIGO Livingston observatory, P.O. Box 940, Livingston, Louisiana, 70754, USA \\ LIGO-P070093-00-Z
}

\begin{abstract}
We describe a coherent network algorithm for detection and reconstruction of gravitational wave bursts. The algorithm works for two and more arbitrarily aligned detectors and can be used for both all-sky and triggered burst searches. We describe the main components of the algorithm, including the time-frequency analysis in wavelet domain, construction of the likelihood time-frequency maps, the identification and selection of burst events.

PACS numbers: $04.80 . \mathrm{Nn}, 07.05 . \mathrm{Kf}, 95.30 . \mathrm{Sf}, 95.85 . \mathrm{Sz}$
\end{abstract}

\section{Introduction}

Coherent network analysis is addressing the problem of detection and reconstruction of gravitational waves (GW) with networks of detectors. It has been extensively studied in the literature [1, 2, 3, 4, 5, 6] in application to detection of bursts signals, which may be produced by numerous gravitational wave sources in the Universe [7, 8, 9, 10, 11, 12, 13, 14, 15]. In coherent methods, a statistic is built up as a coherent sum over detector responses. In general, it is expected to be more optimal (better sensitivity at the same false alarm rate) than the detection statistics of the individual detectors that make up the network. Also coherent methods provide estimators for the GW waveforms and the source coordinates in the sky.

The method we present (called coherent WaveBurst) is significantly different from the traditional burst detection methods. Unlike coincident methods [16, 17, 18], which first identify events in individual detectors by using an excess power statistic and than require coincidence between detectors, the coherent WaveBurst method combines all data streams into one coherent statistic constructed in the framework of the constrained maximum likelihood analysis [4]. Such an approach has significant advantages over the coincident methods. First, the sensitivity of the method is not limited by the least sensitive detector in the network. In the coherent WaveBurst method the detection is based on the maximum likelihood ratio statistic which represents the total signal-to-noise ratio of the GW signal detected in the network. Second, other coherent statistics, such as the null stream and the network correlation coefficient can be constructed to distinguish genuine GW signals from the environmental and instrumental artifacts. Finally, the source coordinates of the GW waveforms can be reconstructed. 


\section{Coherent analysis}

The coherent WaveBurst pipeline (cWB) uses a method, for a coherent detection and reconstruction of burst signals, based on the use of the likelihood ratio functional [4]. For a general case of Gaussian quasi-stationary noise it can be written in the wavelet (time-frequency) domain as

$$
\mathcal{L}=\sum_{k=1}^{K} \sum_{i, j=1}^{N}\left(\frac{w_{k}^{2}[i, j]}{\sigma_{k}^{2}[i, j]}-\frac{\left(w_{k}[i, j]-\xi_{k}[i, j]\right)^{2}}{\sigma_{k}^{2}[i, j]}\right),
$$

where $K$ is the number of detectors in the network, $w_{k}[i, j]$ is the sampled detector data (time $i$ and frequency $j$ indices run over some time-frequency area of size $N$ ) and $\xi_{k}[i, j]$ are the detector responses. Note, we omit the term $1 / 2$ in the conventional definition of the likelihood ratio. The detector noise is characterised by its standard deviation $\sigma_{k}[i, j]$, which may vary over the time-frequency plane. The detector responses are written in the standard notations

$$
\xi_{k}[i, j]=F_{+k} h_{+}[i, j]+F_{\times k} h_{\times}[i, j],
$$

where $F_{+k}(\theta, \phi), F_{\times k}(\theta, \phi)$ are the detector antenna patterns (depend upon source coordinates $\theta$ and $\phi$ ) and $h_{+}[i, j], h_{\times}[i, j]$ are the two polarisations of the gravitational wave signal in the wave frame. Since the detector responses $\xi_{k}$ are invariant with respect to the rotation around z-axis in the wave frame, the polarization angle is included in the definition of $h_{+}$and $h_{\times}$. The GW waveforms, $h_{+}$and $h_{\times}$, are found by variation of $\mathcal{L}$. The maximum likelihood ratio statistic is obtained by substitution of the solutions into the functional $\mathcal{L}$. The waveforms in the time domain are reconstructed from the inverse wavelet transformation. Below, for convenience we introduce the data vector $\mathbf{w}[i, j]$ and the antenna pattern vectors $\mathbf{f}_{+}[i, j]$ and $\mathbf{f}_{\times}[i, j]$

$$
\begin{aligned}
& \mathbf{w}[i, j]=\left(\frac{w_{1}[i, j]}{\sigma_{1}[i, j]}, . ., \frac{w_{K}[i, j]}{\sigma_{K}[i, j]}\right) \\
& \mathbf{f}_{+(\times)}[i, j]=\left(\frac{F_{1+(\times)}}{\sigma_{1}[i, j]}, . ., \frac{F_{K+(\times)}}{\sigma_{K}[i, j]}\right)
\end{aligned}
$$

Further in the text we omit the time-frequency indices and replace the sum $\sum_{i, j=1}^{N}$ with $\sum_{\Omega_{T F}}$, where $\Omega_{T F}$ is the time-frequency area selected for the analysis.

The likelihood functional (Eq.1) can be written in the form $\mathcal{L}=\mathcal{L}_{+}+\mathcal{L}_{\times}$:

$$
\begin{aligned}
& \mathcal{L}_{+}=\sum_{\Omega_{T F}}\left[\left(\mathbf{w} \cdot \mathbf{f}_{+}\right) h_{+}-\frac{1}{2}\left|\mathbf{f}_{+}\right|^{2} h_{+}^{2}\right], \\
& \mathcal{L}_{\times}=\sum_{\Omega_{T F}}\left[\left(\mathbf{w} \cdot \mathbf{f}_{\times}\right) h_{\times}-\frac{1}{2}\left|\mathbf{f}_{\times}\right|^{2} h_{\times}^{2}\right],
\end{aligned}
$$

where the antenna pattern vectors $\mathbf{f}_{+}$and $\mathbf{f}_{\times}$are defined in the Dominant Polarisation wave Frame (DPF) [4]. In this frame the antenna pattern vectors are orthogonal to each other: $\left(\mathbf{f}_{+} \cdot \mathbf{f}_{\times}\right)=\mathbf{0}$. The estimators of the GW waveforms are the solutions of the 
equations

$$
\begin{aligned}
& \left(\mathbf{w} \cdot \mathbf{f}_{+}\right)=\left|\mathbf{f}_{+}\right|^{2} h_{+}, \\
& \left(\mathbf{w} \cdot \mathbf{f}_{\times}\right)=\left|\mathbf{f}_{\times}\right|^{2} h_{\times} .
\end{aligned}
$$

Note, the norms $\left|\mathbf{f}_{+}\right|^{2}$ and $\left|\mathbf{f}_{\times}\right|^{2}$ characterise the network sensitivity to the $h_{+}$and $h_{\times}$ polarisations respectively.

\subsection{Likelihood regulators}

As first shown in [4], there is a specific class of constraints (often called regulators), which arise from the way the network responds to a generic GW signal. A classical example is a network of aligned detectors where the detector responses $\xi_{k}$ are identical. In this case the algorithm can be constrained to search for an unknown function $\xi$ rather than for two GW polarisations $h_{+}$and $h_{\times}$, which span a much larger parameter space. Note, that in this case $\left|\mathbf{f}_{\times}\right|^{2}=0$, the Equation 8 is ill-conditioned and the solution for the $h_{\times}$waveform can not be found. The regulators are important not only for aligned detectors, but also for networks of miss-aligned detectors, for example, the LIGO and Virgo network [20, 21]. Depending on the source location, the network can be much less sensitive to the second GW component $\left(\left|\mathbf{f}_{\times}\right|^{2}<<\left|\mathbf{f}_{+}\right|^{2}\right)$ and the $h_{\times}$waveform may not be reconstructed from the noisy data.

In the coherent WaveBurst analysis we introduce a regulator by changing the norm of the $\mathbf{f}_{\times}$vector

$$
\left|\mathbf{f}_{\times}^{\prime}\right|^{2}=\left|\mathbf{f}_{\times}\right|^{2}+\delta
$$

where $\delta$ is a parameter. This is equivalent to adding one more dummy detector to the

network with the antenna patterns $f_{+, K+1}=0, f_{\times, K+1}=\sqrt{\delta}$ and zero detector output $\left(x_{K+1}=0\right)$. In this case, the regulator preserves the orthogonality of the vectors $\mathbf{f}_{+}$and $\mathbf{f}_{\times}^{\prime}$ and the maximum likelihood statistic is written as

$$
L_{\max }=\sum_{\Omega_{T F}}\left[\frac{\left(\mathbf{w} \cdot \mathbf{f}_{+}\right)^{2}}{\left|\mathbf{f}_{+}\right|^{2}}+\frac{\left(\mathbf{w} \cdot \mathbf{f}_{\times}^{\prime}\right)^{2}}{\left|\mathbf{f}_{\times}^{\prime}\right|^{2}}\right]=\sum_{\Omega_{T F}}\left[\left(\mathbf{w} \cdot \mathbf{e}_{+}\right)^{2}+\left(\mathbf{w} \cdot \mathbf{e}_{\times}^{\prime}\right)^{2}\right],
$$

where the $\mathbf{e}_{+}$and $\mathbf{e}_{\times}^{\prime}$ are unit vectors. Depending on the value of the parameter $\delta$ different statistics can be generated, for example:

- $\delta=0$ - standard likelihood,

- $\delta=\infty$ - hard constraint likelihood.

\subsection{Reconstruction of $G W$ waveforms}

The GW waveforms are given by the solutions of the likelihood functional Eq 5,6. For the first GW component the solution is

$$
h_{+}=\frac{\left(\mathbf{w} \cdot \mathbf{f}_{+}\right)}{\left|\mathbf{f}_{+}\right|^{2}} \text {. }
$$


When the regulator is introduced it affects the solution for the second GW component. In this case we look for such a solution, which gives the second term of the likelihood statistic $L_{\max }$, when the solution is substituted into the likelihood functional. Namely, we solve the equation

$$
2\left(\mathbf{w} \cdot \mathbf{f}_{\times}\right) h_{\times}-\left|\mathbf{f}_{\times}\right|^{2} h_{\times}^{2}-\frac{\left(\mathbf{w} \cdot \mathbf{f}_{\times}^{\prime}\right)^{2}}{\left|\mathbf{f}_{\times}^{\prime}\right|^{2}}=0 .
$$

Out of two possible solutions the following one is selected

$$
h_{\times}=\frac{\left(\mathbf{w} \cdot \mathbf{f}_{\times}\right)}{\left|\mathbf{f}_{\times}^{\prime}\right|^{2}}\left(1+\sqrt{1-\frac{\left|\mathbf{f}_{\times}\right|^{2}}{\left|\mathbf{f}_{\times}^{\prime}\right|^{2}}}\right)^{-1} .
$$

In case of aligned detectors $\left(\left|f_{\times}\right|=0\right)$ this equation results in a trivial solution $h_{\times}=0$.

\section{Data analysis algorithms}

In this section we describe the algorithms used in the coherent WaveBurst pipeline. They include: wavelet transformation, conditioning of input data, construction of time delay filters, and generation of coherent triggers.

\subsection{Wavelet transformation}

The discrete wavelet transformations (DWT) are applied to discrete data and produce discrete wavelet series $w[i, j]$, where $j$ is the scale index and $i$ is a time index. Applied to time series, the DWT maps data from time domain to the wavelet domain. All DWTs used in cWB have critical sampling when the output data vector has the same size as the input data vector.

Wavelet series give a time-scale representation of data where each wavelet scale can be associated with a certain frequency band of the initial time series. Therefore the wavelet time-scale spectra can be displayed as a time-frequency (TF) scallogram, where the scale is replaced with the central frequency $f$ of the band. The time series sampling rate $R$ and the scale number $j$ determine the time resolution $\Delta t_{j}(R)$ at this scale. The DWT preserves the time-frequency volume of the data samples, which is equal to $1 / 2$ for the input time series. Therefore the frequency resolution $\Delta f_{j}$ is defined as $1 /\left(2 \Delta t_{j}\right)$ and determines the data bandwidth at the scale $j$. For optimal localisation of the GW energy on the TF plane, the cWB analysis is performed at several time-frequency resolutions.

The time-frequency resolution defined above should be distinguished from the intrinsic time-frequency resolution of the wavelet transformation, which defines the spectral leakage between the wavelet sub-bands and depends on the length of the wavelet filter. To reduce spectral leakage we use Meyers wavelets for which long filters can be easily constructed [22]. As shown in Figure 1, it allows us much better localization of the burst energy on the time-frequency plane than Symlet60 wavelets used for the LIGO S2-S4 analysis [23, 24]. The disadvantage of the Meyer filters is that for the local support they have to be truncated. As the result, the Meyer wavelets are approximately 


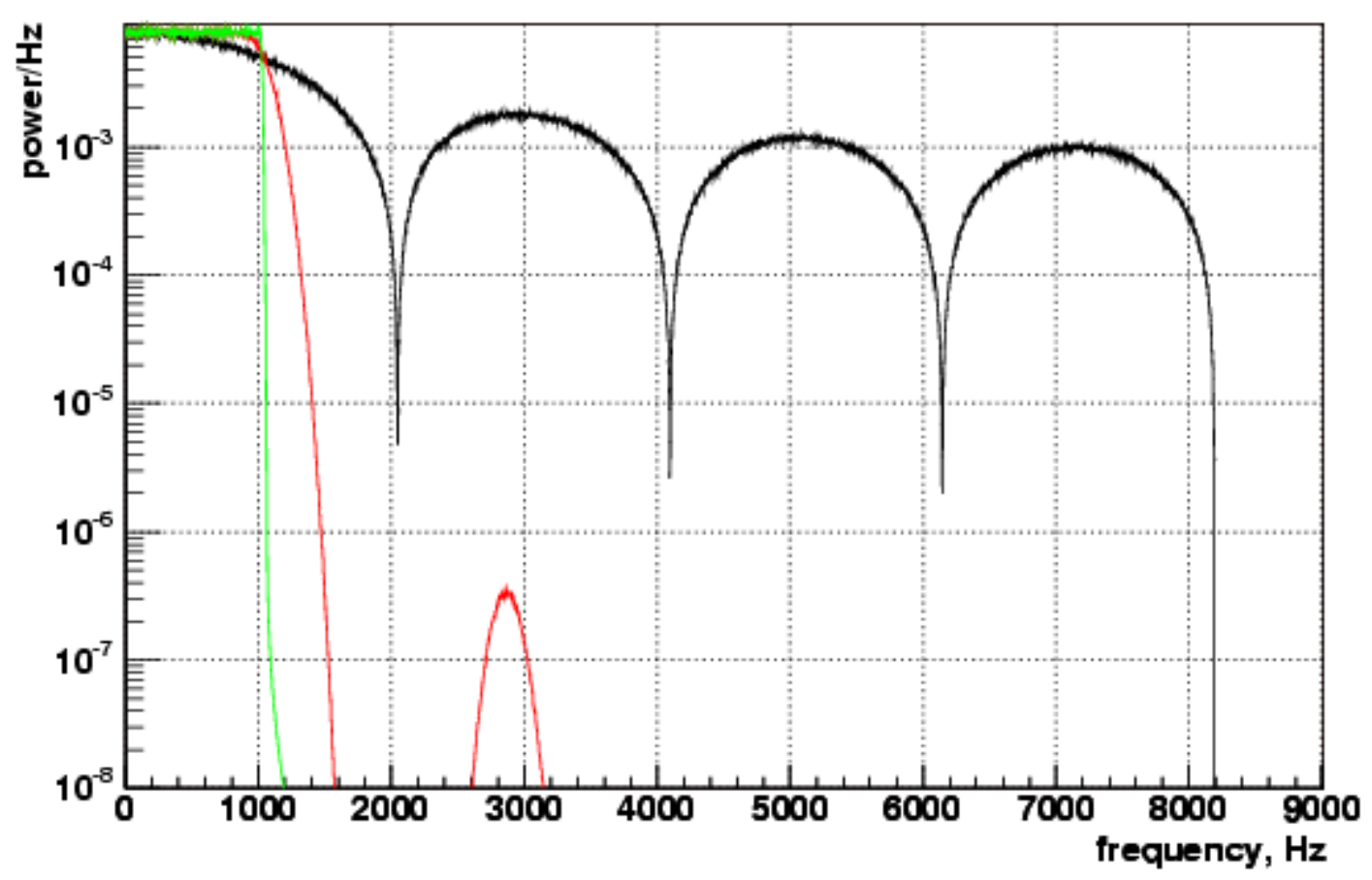

Figure 1. Comparison of spectral leakage from the first (low) frequency band $0-1024 \mathrm{~Hz}$ to the high frequency bands for Haar (black), Symlet60 (red) and Meyer1024 (green) wavelets after three wavelet decomposition steps.

orthonormal. From the other side the Meyer filters can be constructed so that the Parseval identity holds with better then $0.01 \%$ accuracy, which is more than adequate for the analysis.

\subsection{Linear prediction error filter}

The linear prediction error (LPE) filters are used to remove "predictable" components from an input time series. Usually they are constructed and applied in the time domain. In this case the output of the LPE filter is a whitened time series. The LPE filters can be also used in the wavelet domain. For construction of the LPE filters we follow the approach described in [26]. The symmetric LPE filters can be constructed from the backward and forward LPE filters by using classical Levinson algorithm, or the split lattice algorithm.

Since each wavelet layer is a time series, rather then applying the LPE filter to a time series $x(t)$, one can perform a wavelet decomposition $x(t) \rightarrow w(f, t)$ first, and then construct and apply the LPE filter $F(f)$ individually to each wavelet layer. A set of filters $F(f)$ removes predictable components (like lines) in the wavelet layers producing data $w^{\prime}(t)$. The filtered time series $x^{\prime}(t)$ can be reconstructed from $w^{\prime}(t)$ with the inverse wavelet transformation. An example PSD of the filtered segment of S4 data is shown 
in Figure 2, As one can see, when applied in wavelet domain the LPE filter removes spectral lines but preserves the power spectral density of the noise floor.

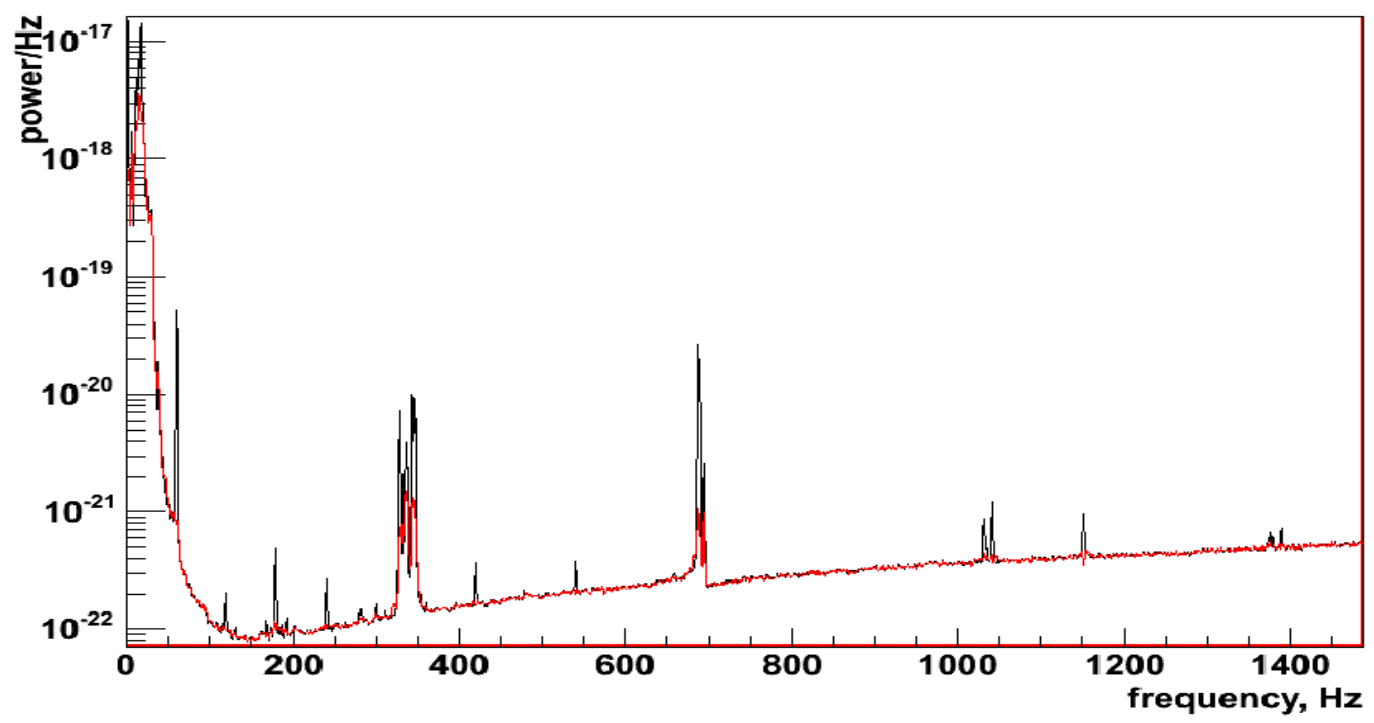

Figure 2. Power spectra of original (black) and LPE filtered (red) noise of the Hanford $4 \mathrm{k}$ detector.

\subsection{Time delay filters in the wavelet domain}

The likelihood method requires calculation of the inner products $\left\langle x_{n}\left(\tau_{n}\right), x_{m}\left(\tau_{m}\right)\right\rangle$, where the data streams are shifted in time to take into account the GW signal time delay between the detectors $n$ and $m$. The time delay $\tau_{n}-\tau_{m}$ in turn, depends on the source coordinates $\theta$ and $\phi$.

In time domain it is straightforward to account for the time delays. However for colored detector noise it is preferable to calculate the maximum likelihood statistics in the Fourier or wavelet (time-frequency) domains. In the wavelet domain one needs to calculate the inner products $\left\langle w_{n}\left(\tau_{n}\right), w_{m}\left(\tau_{m}\right)\right\rangle$. The delayed amplitudes can be calculated from the original amplitudes (before delay) with the help of the time delay filter $D_{k l}(\tau)$

$$
w_{n, m}(i, j, \tau)=\sum_{k l} D_{k l}(\tau, j) w_{n, m}(i+k, j+l),
$$

where $k$ and $l$ are the local TF coordinates with respect to the TF location $(i, j)$. The delay filters are constructed individually for each wavelet layer, which is indicated with the index $j$.

The construction of time delay filters is related to the decomposition of the sampled wavelet functions $\Psi_{j}(t+\tau)$ in the basis of the non-shifted wavelet functions $\Psi_{j}(t)$. The filter construction procedure can be described in the following steps:

- create a wavelet series with only one coefficient at the TF location $(i, j)$ set to unity, 
- apply the inverse wavelet transformation reconstructing $\Psi_{j}(t)$ in time domain,

- shift $\Psi_{j}(t)$ by delay time $\tau$ and perform wavelet decomposition of $\Psi_{j}(t+\tau)$,

- the resulting wavelet amplitudes at the TF locations $(i+k, j+l)$ give the delay filter coefficients $D_{k l}(\tau, j)$ for the wavelet layer $j$.

The length of the filter is determined by the requirement on the acceptable energy loss when the time delay filter is applied. The fractional energy loss is

$$
\epsilon_{K}=1-\sum_{K} D_{k l}^{2}
$$

where the sum is calculated over the $K$ most significant coefficients. The selected coefficients are also described by the list of their relative $\mathrm{TF}$ locations $(k, l)$ which should be stored along with the filter coefficients $D_{k l}$. Typically $K$ should be greater then 20 to obtain the fractional energy loss less than $1 \%$.

\subsection{Generation of coherent triggers}

A starting point of any burst analysis is the identification of burst events (triggers). Respectively, this stage of the burst analysis pipeline is called the event trigger generator (ETG). Usually, the ETGs based on the excess power statistics of individual detectors are used in the analyses [16, 17, 18]. Another example of an ETG is the CorrPower algorithm [19], which uses cross-correlation between aligned detector pairs to generate the triggers. The likelihood statistic used in the coherent WaveBurst utilizes both the excess power and the cross-correlation terms.

3.4.1. Likelihood time-frequency maps In general the likelihood functional is calculated as a sum over the data samples selected for the analysis (see Eq.1). The number of terms in the sum depends on the selected $\mathrm{TF}$ area in the wavelet domain. When the sum consists of only one term, one can write the likelihood functional for a given TF location and point in the sky

$$
\mathcal{L}_{p}(i, j, \theta, \phi)=|\mathbf{w}|^{2}-\left|\mathbf{w}-\mathbf{f}_{+} h_{+}-\mathbf{f}_{\times} h_{\times}\right|^{2} .
$$

Since the entire likelihood approach is applicable to the functional above, one can solve the variation problem and find the maximum likelihood statistics $L_{p}(\theta, \phi)$. They can be maximized over the source coordinates $\theta$ and $\phi$, resulting in the statistics

$$
L_{m}(i, j)=\max _{\theta, \phi}\left\{L_{p}(i, j, \theta, \phi)\right\} .
$$

Calculated as a function of time and frequency, it gives a likelihood time-frequency (LTF) map. Figure 3 shows an example of the LTF map for a segment of the S4 data.

A single data sample in the map is called the LTF pixel. It is characterized by its TF location $(i, j)$ and by the arrays of wavelet amplitudes $w_{k}\left(i, j, \tau_{k}(\theta, \phi)\right)$, which are used to construct the likelihood statistics $L_{p}$.

$\ddagger$ For definition of vectors $\mathbf{w}, \mathbf{f}_{+}$, and $\mathbf{f}_{\times}$see Eq 34 


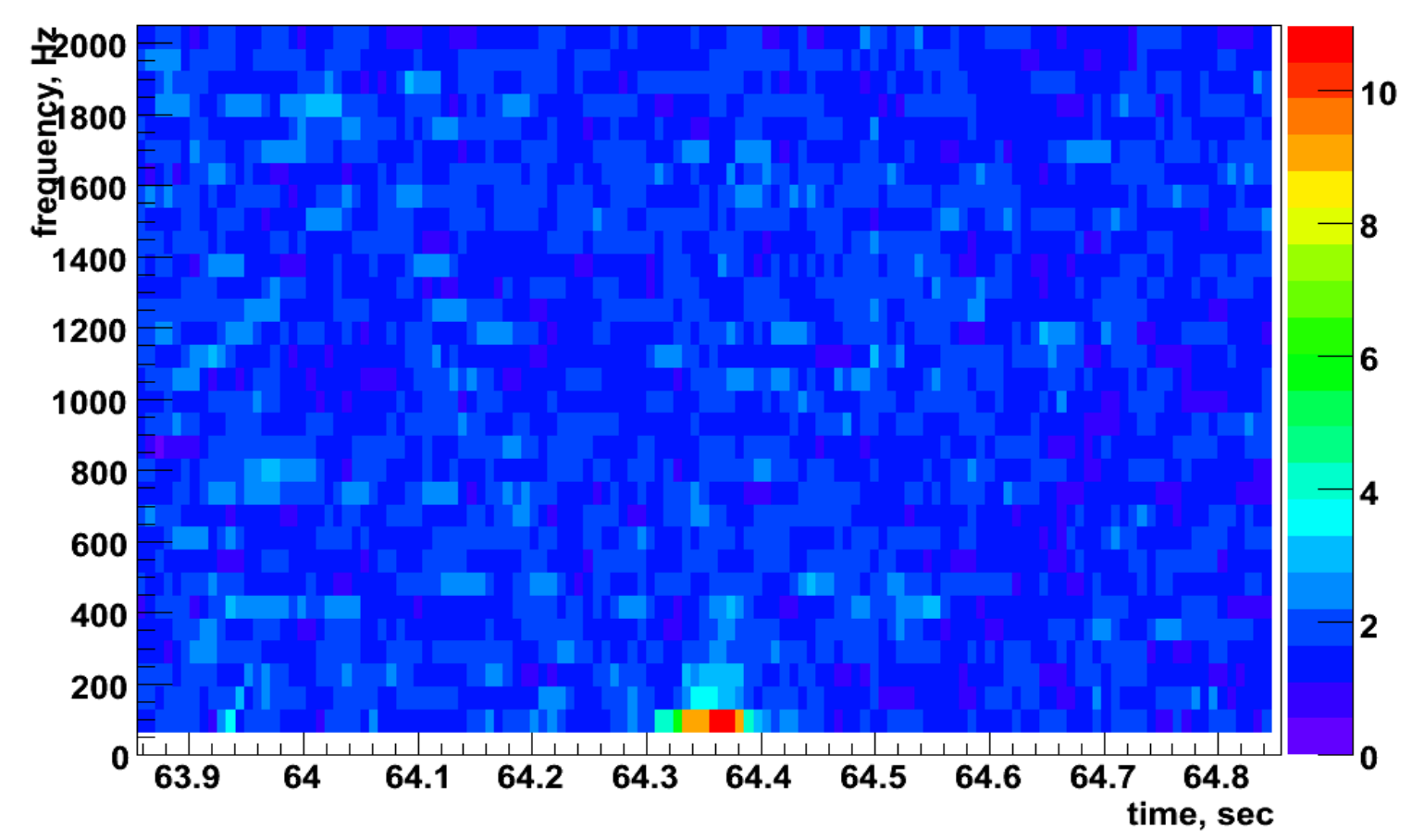

Figure 3. Example of the likelihood time-frequency map for a magnetic glitch in the S4 L1xH1xH2 data.

3.4.2. Coherent triggers The statistic $L_{m}$ has a meaning of the maximum possible energy detected by the network at a given TF location. By selecting the values of $L_{m}$ above some threshold, one can identify groups of the LTF pixels (coherent trigger) on the time-frequency plane. A coherent trigger is defined for the entire network, rather than for the individual detectors. Therefore, further in the text we reserve a name "cluster" for a group of pixels selected in a single detector and refer to a group of the LTF pixels as a coherent or network trigger.

After the coherent triggers are identified, one has to reconstruct the parameters of the GW bursts associated with the triggers, including the reconstruction of the source coordinates, the two GW polarisations, the individual detector responses and the maximum likelihood statistics of the triggers. The likelihood of reconstructed triggers is calculated as

$$
\mathcal{L}_{c}(\theta, \phi)=\sum_{i j} \mathcal{L}_{p}(i, j, \theta, \phi)
$$

where the sum is taken over the LTF pixels in the trigger. The maximum likelihood statistic $L_{\max }$ is obtained by variation of $L_{c}$ over $\theta$ and $\phi$. Unlike for $L_{p}$, which is calculated for a single LTF pixel, the $L_{\max }$ is calculated simultaneously for all LTF pixels forming the coherent trigger. 


\section{Selection of coherent triggers}

When the detector noise is Gaussian and stationary, the maximum likelihood $L_{\max }$ is the only statistic required for detection and selection of the GW events. In this case the false alarm and the false dismissal probabilities are controlled by the threshold on $L_{\text {max }}$. The real data however, is contaminated with the instrumental and environmental glitches and additional selection cuts should be applied to distinguish genuine GW signals [6, 25]. Such selection cuts test the consistency of the reconstructed responses in the detectors. In the coherent WaveBurst method the consistency test is based on the coherent statistics constructed from the elements of the likelihood and the null matrices.

The likelihood matrix $L_{n m}$ is obtained from the likelihood quadratic form (see $\mathrm{Eq}, 10$

$$
L_{\max }=\sum_{n m} L_{n m}=\sum_{n m}\left[\left\langle w_{n} w_{m} e_{+n} e_{+m}\right\rangle+\left\langle w_{n} w_{m} e_{\times n}^{\prime} e_{\times m}^{\prime}\right\rangle\right] .
$$

where $n$ and $m$ are the detector indexes. The diagonal (off-diagonal) terms of the matrix $L_{m n}$ describe the reconstructed normalized incoherent (coherent) energy. The sum of the off-diagonal terms is the coherent energy $E_{\text {coh }}$ detected by the network. The coherent terms can be used to construct the correlation coefficients:

$$
r_{n m}=\frac{L_{n m}}{\sqrt{L_{n n} L_{m m}}} .
$$

which represent Pearson's correlation coefficients in the case of aligned detectors. We use the coefficients $r_{n m}$ to construct the reduced coherent energy

$$
e_{\mathrm{coh}}=\sum_{n m} L_{n m}\left|r_{n m}\right|, \quad n \neq m \text {. }
$$

which provides one of the most efficient selection cuts for rejection of the incoherent background events.

The null matrix represents the normalized energy of the reconstructed noise

$$
N_{n m}=E_{n m}-L_{n m},
$$

where $E_{n m}$ is the diagonal matrix of the normalized energy in the detectors: $E_{n n}=\left\langle x_{n}^{2}\right\rangle$. To distinguish genuine GW signals from the instrumental and invironmental glitches we introduce the network correlation coefficients

$$
C_{\text {net }}=\frac{E_{\text {coh }}}{N_{\text {ull }}+\left|E_{\mathrm{coh}}\right|}, \quad c_{\text {net }}=\frac{e_{\mathrm{coh}}}{N_{\mathrm{ull}}+\left|e_{\mathrm{coh}}\right|}
$$

where $N_{\text {ull }}$ is a sum of all elements of the null matrix, which represents the total

energy in the null stream. Usually for glitches little coherent energy is detected and the reconstructed detector responses are inconsistent with the detector outputs which results in the large null energy. Therefore the correlation coefficients $C_{\text {net }}$ and $c_{\text {net }}$ can be used for a signal consistency test which effectively compares the null energy with the coherent energy. This is much safer consistency test than the null stream veto [25] where the null energy is compared with the estimated noise energy. In any realistic data analysis there is always some residual energy left in the null stream. Therefore for 
strong gravitational waves the energy of the residual signal can be much larger than the noise energy that may result in the false rejection of the GW signals. This is not the case for the veto cut based on the $C_{\text {net }}$ and $c_{\text {net }}$.

\section{Summary}

In the paper we discussed how the coherent network algorithms are constructed for burst searches. We found it convenient to construct coherent burst searches in the time-frequency (wavelet) domain, which requires construction of time delay filters. For detection of burst signals we combine output of all detectors into one coherent statistic - likelihood, which represents the total signal-to-noise ratio of the signal detected in the network. To distinguish genuine GW signals from the instrumental and environmental glitches we introduced several coherent statistics constructed from the elements of the likelihood and null matrices. We do not discuss the performance of the method in this paper, however, numerous studies of the method with different sets of LIGO and Virgo data have been performed. It was found, in general, that the method has better performance than the burst algorithm used for the published analysis of the LIGO data [16, 20, 21]. The results of these studies will be presented in subsequent papers.

\section{Acknowledgments}

We thank Keith Riles, Michele Zanolin and Brian O'Reilly for detailed discussions and review of the algorithm and suggestions which significantly improved its performance. This work was supported by the US National Science Foundation grant PHY-0555453 to the University of Florida, Gainesville.

\section{References}

[1] Y. Gursel and M. Tinto, Phys. Rev. D 40, 3884 (1989)

[2] E. E. Flanagan and S. A. Hughes, Phys. Rev. D 57, 4577 (1998)

[3] N. Arnaud et al, Phys. Rev. D 68, 102001 (2003)

[4] S.Klimenko, S.Mohanty, M.Rakhmanov, G.Mitselmakher, Phys. Rev. D 72, 122002 (2005).

[5] M. Rakhmanov Class. Quantum Grav. 23 S673 (2006)

[6] S. Chatterji et al, Phys.Rev. D 74082005 (2006)

[7] T. Zwerger and E. Mueller, Astron. Astrophys, 320, 209 (1997)

[8] H. Dimmelmeier, J. A. Font and E. Mueller, Astron. Astrophys, 393, 523 (2002)

[9] C. Ott et al, Astrophys. J., 600, 834 (2004)

[10] M. Shibata and Y. I. Sekiguchi, Phys. Rev. D 69, 084024 (2004)

[11] E. E. Flanagan and S. A. Hughes, Phys. Rev. D 57, 4535 (1998)

[12] J. Baker et al, Phys. Rev. D 73, 104002 (2006)

[13] F. Pretorius, Phys. Rev. Lett. 95, 121101 (2005)

[14] M. Campanelli et al, Phys. Rev. Lett. 96, 111101 (2006)

[15] P. Meszaros, Rept. Prog. Phys. 69, 2259-2322 (2006)

[16] S. Klimenko and G. Mitselmakher, Wavelet method for GW burst detection Class. Quantum Grav. 21, S1819 (2004). Class. Quantum Grav. 21, S1685 (2004). 
[17] S. Chatterji, L. Blackburn, G. Martin, E. Katsavounidis, Multiresolution techniques for the detection of gravitational-wave bursts. Class. Quantum Grav. 21, S1809 (2004).

[18] J.W.C. McNabb et al, arXiv:gr-qc/0404123 v1 29, April 2004

[19] L. Cadonati, Coherent waveform consistency test for LIGO burst candidates. Class. Quantum Grav. 21, S1695 (2004).

[20] B. Abbott et al, (The LIGO Scientific Collaboration), Class. Quantum Grav. 23, S51-S56 (2006)

[21] F. Acernese et al, (The Virgo Collaboration), Class. Quantum Grav. 23, S63-S69 (2006)

[22] B. Vidakovic, Statistical modeling by wavelets, 1999.

[23] B. Abbot et al, Phys. Rev. D 72, (2005) 062001

[24] B. Abbot et al, Class. Quantum Grav. 24 5343-5369 (2007)

[25] L. Wen and B. F. Schutz,Coherent network detection of gravitational waves: the redundancy veto, Class. Quantum Grav. 22, S1321 (2005).

[26] P. Delsarte and Y. Genin, On the Splitting of classical algorithms in Linear prediction theory, IEEE, ASSP-35, 1987 